\title{
An Empirical Analysis of Director Mingjun Zhao's Experience in Treating Chronic Heart Failure
}

\author{
Zijuan He ${ }^{1}$, Mingjun Zhao' ${ }^{1,2 *}$ \\ 1Shaanxi University of Chinese Medicine, Xianyang 712046, Shaanxi Province, China \\ ${ }^{2}$ The Affiliated Hospital of Shaanxi University of Chinese Medicine, Xianyang 712046, Shaanxi Province, China \\ *Corresponding author: Mingjun Zhao, zmj7125@163.com
}

\begin{abstract}
With the aggravation of an aging population, the prevalence of chronic heart failure is increasing. As a famous traditional Chinese medicine practitioner in Shaanxi Province, Mingjun Zhao is amicable at using traditional Chinese medicine to treat chronic heart failure. He believes that the pathogenesis of the condition is mainly due to the lack of heart Yang, deficiency of heart Qi, as well as the combination of water and blood stasis. Therefore, in treating this condition, it is essential to warm and nourish heart Yang, replenish Qi to benefit water, and promote blood circulation to remove blood stasis. Based on the standardized treatment used in western medicine, traditional Chinese medicine should be reasonably and accurately combined with it as a drug pair into a prescription, thus highlighting the advantages of the combination of traditional Chinese medicine in the treatment of chronic heart failure to a great extent.
\end{abstract}

Keywords: Chronic heart failure; Traditional Chinese medicine; Drug pairing; Mingjun Zhao

Publication date: November 2021; Online publication: November 30, 2021

\section{Introduction}

Heart failure is under the categories of "heart distention," "cardiac edema," "heart impediment," and "heart cough" in traditional Chinese medicine ${ }^{[1]}$. In the treatment of chronic heart failure, Mingjun Zhao, a chief physician, follows the thinking of traditional Chinese medicine. He is familiar with the compatibility of nature, taste, and efficacy of traditional Chinese medicine. Through the reasonable matching of drugs, he maximizes the treatment effect while minimizes the side effects. Being a student of Mingjun Zhao, the author has benefited a lot by observing the chief physician through his clinical practice, especially in the treatment of chronic heart failure using traditional Chinese medicine. This article discusses Mingjun Zhao's experience in treating chronic heart failure.

\section{Academic view}

Mingjun Zhao believes that chronic heart failure is rooting vacuity and tipping repletion in the disease occurrence and development. Blood stasis and water-drinking are regarded as tipping repletion. If tonic medicine is heavily used, it would be too greasy, and evil Qi would stay in the body. If the medicine is used only for the purpose of attacking evil but neglects the healthy Qi of the body, the healthy Qi of the body would become weaker. Therefore, in the treatment of chronic heart failure, Mingjun Zhao believes that traditional Chinese medicine should be used to make Qi vacuity tonic, vacuous Yang warm, water-drinking subside, and blood stasis melt, so as to achieve the goal. 


\section{Research methods}

The medical records of patients with chronic heart failure listed as the first diagnosis in Zhao Mingjun's clinic in the recent 5 years were reviewed. The information of 190 cases who had sought medical intervention repeatedly for chronic heart failure but eventually chose traditional Chinese medicine decoction prescribed by Zhao Mingjun or fried-free granules given in the hospital were screened and entered into Excel 2016. The information was then imported into SPSS 25.0, which included the patients' name, gender, age, and drug. The commonly used drug pairs were obtained by cluster analysis, and the commonly used amount of drugs were obtained by frequency statistical analysis.

\section{Results}

The results showed that there are four pairs of drugs commonly used by Zhao Mingjun for treating chronic heart failure. These four pairs of drugs are discussed below.

\subsection{Radix Astragali seu Hedysari and Ramulus Cinnamomi}

Radix Astragali seu Hedysari has the effects of tonifying Qi and lifting Yang as well as inducing diuresis to alleviate edema. Studies have shown that Radix Astragali seu Hedysari can significantly improve the antioxidant capacity of cardiomyocytes and tissues in the event of heart failure ${ }^{[2]}$. In addition, it can inhibit the apoptosis of cardiomyocytes, protect the myocardium, alleviate ischemia and hypoxia, as well as improve ventricular remodeling. Ramulus Cinnamomi has the effects of warming meridians, dredging meridians, assisting Yang, and promoting Qi. Studies have shown that cinnamaldehyde contained in Ramulus Cinnamomi can relax central and peripheral blood vessels, promote blood flow, and improve cardiac function ${ }^{[3]}$. Zhao Mingjun believes that the two drugs can alleviate edema, arrhythmia, and sleepiness caused by chronic heart failure. The common dosage of Radix Astragali seu Hedysari is 30 grams and that of Ramulus Cinnamomi is 10 grams.

\subsection{Radix Codonopsis and Rhizoma Atractylodis Macrocephalae}

Radix Codonopsis has the effects of tonifying Qi and nourishing blood. The results from a study conducted by Qun Gao and other researchers showed that in chronic heart failure, where the peak value of calcium ion decreases, and the systolic and diastolic function of the heart fails, Radix Codonopsis can be used to improve the systolic function of damaged cardiomyocytes ${ }^{[4]}$. Rhizoma Atractylodis Macrocephalae has the effects of supplementing Qi, strengthening spleen, drying dampness, and promoting water. It can also improve the state of the cardiovascular system. Experimental studies have shown that atractylenolide-I can significantly reduce the effect of acetic acid on vascular permeability in hypoxic mice ${ }^{[5]}$. Therefore, in chronic heart failure, Rhizoma Atractylodis Macrocephalae can protect myocardium, resist oxidation, tranquilize, and allay excitement. Zhao Mingjun believes that patients with chronic heart failure would have spleen and stomach deficiencies for a long time, resulting in Qi and blood deficiencies. Radix Codonopsis combined with Rhizoma Atractylodis Macrocephalae can strengthen the spleen, tonify Qi, and activate the blood. The dosage for Radix Codonopsis is usually 15 grams and that of Rhizoma Atractylodis Macrocephalae is 10 grams.

\subsection{Semen Plantaginis and Radix Achyranthis Bidentatae}

Semen Plantaginis has the effects of inducing diuresis for treating strangury, draining dampness, and eliminating phlegm. Pharmacological studies have concluded that Semen Plantaginis has many effects, such as diuresis, immune regulation, antioxidation, blood lipid reduction, anti-inflammatory effect, anti- 
viral effect, hypoglycemic effect, and anti-aging ${ }^{[6]}$. Radix Achyranthis Bidentatae has the effects of removing blood stasis and dredging meridians, ensuring a proper downward flow of the blood as well as inducing diuresis for treating strangury. Zhao Mingjun believes that Radix Achyranthis bidentatae can disinhibit water, free strangury, activate blood, and remove stasis; in combination with Semen Plantaginis, they can activate blood, remove stasis, induce diuresis, eliminate rheum, as well as dredge and regulate water passage. They can be used to treat ascites and edema of both lower limbs caused by chronic heart failure. The common dosage of Semen Plantaginis is 15 grams and that of Radix Achyranthis Bidentatae is 15 grams.

\subsection{Radix Salviae Miltiorrhizae and Flos Carthami}

Radix Salviae Miltiorrhizae is good for dredging blood vessels. It can also tranquilize, clear away heart fire, as well as treat palpitations and insomnia. Pharmacological studies have shown that Radix Salviae Miltiorrhizae has the effect of regulating hemodynamics in the blood circulation system ${ }^{[7]}$. On the other hand, Flos Carthami can be used for treating chest stuffiness and pain caused by blood stasis. Carthamin yellow, contained in Flos Carthami, can expand the coronary arteries, improve blood supply, reduce oxygen consumption of cardiomyocytes, and regulate microcirculation, so as to fight against myocardial ischemia ${ }^{[8]}$. At the same time, studies have shown that Radix Salviae Miltiorrhizae combined with safflower can increase coronary blood flow, nourish myocardium, regulate platelet function, promote anti-atherosclerosis, improve myocardial metabolism, reduce myocardial oxygen consumption, and alleviate myocardial damage caused by ischemia and hypoxia ${ }^{[9]}$. Zhao Mingjun believes that the combination of Radix Salviae Miltiorrhizae and Flos Carthami can enhance the efficacy of removing blood stasis, dredging meridians, and relieving pain. At the same time, Radix Salviae Miltiorrhizae has a calming effect and can be used to treat insomnia; in addition, it can be used to treat patients with chest pain, anxiety, and other symptoms. The common dosage of Radix Salviae Miltiorrhizae is 15 grams and that of Flos Carthami is 10 grams.

\section{Conclusion}

Mingjun Zhao believes that the two pathological factors, blood stasis and water stagnation, play a part in the whole process of chronic heart failure. In order to prevent excess-resulted deficiency and benefit deficiency due to excess, it is ingenious to cut off such a vicious cycle by promoting blood circulation to remove blood stasis, inducing diuresis, and eliminating rheum. It is a clever way to use the prescription. There are four pairs of drugs commonly used in clinics: Radix Astragali seu Hedysari and Ramulus Cinnamomi; Radix Codonopsis and Rhizoma Atractylodis Macrocephalae; Semen Plantaginis and Radix Achyranthis Bidentatae; Radix Salviae Miltiorrhizae and Flos Carthami. The clinical condition of this disease is complex and changeable; however, Mingjun Zhao's clinical syndrome differentiation and selection of prescriptions had achieved excellent results each time. The selections of prescriptions and drugs by Mingjun Zhao have not been described completely in this article, but this article provides a glimpse while summarizing the experience of a famous traditional Chinese medicine practitioner in treating chronic heart failure, in hope to provide some kind of reference.

\section{Funding}

This research was supported by the Construction Project of Mingjun Zhao Inheritance Studio of Famous Traditional Chinese Medicine (Number: 2019007). 


\section{Disclosure statement}

The authors declare no conflict of interest.

\section{References}

[1] Wu W, 2013, Study on Heart Failure as the Name of Heart Failure in Chinese Medicine. Journal of Traditional Chinese Medicine, 54(10): 891.

[2] Guang P, Xu B, Li Y, et al., 2013, Protective Effect of Astragalus Polysaccharide on DoxorubicinInduced Heart Failure in Rats. Acta Anatomica Sinica, 44(05): 685-688.

[3] Liu P, Zhang LP, 2012, Study on Chemical Constituents and Cardiovascular Pharmacological Effects of Ramulus Cinnamomiand. Liaoning Journal of Traditional Chinese Medicine, 10: 1926-1927.

[4] Gao Q, Li N, Lin Q, 2017, Effects of Radix Astragali seu Hedysari and Radix Codonopsis on Calcium Transients in Cardiomyocytes of Mice with Heart Failure. Journal of Traditional Chinese Medicine, 16: 1408-1411.

[5] Eang H, Yang N, Tan J, et al., 2018, Research Progress on Chemical Constituents, Pharmacological Action and Clinical Application of Rhizoma Atractylodis Macrocephalae. Gansu Medical Journal, 01: 23-26.

[6] Xu S, Xu W, Liang X, et al., 2019, Research Progress on Chemical Constituents and Biological Activities of Semen Plantaginis. Northwest Pharmaceutical Journal, 04: 567-570.

[7] Feng Y, 2017, Modern Pharmacological Research and Clinical Application of Radix Salviae Miltiorrhizae. Clinical Journal of Chinese Medicine, 9(30): 46-47.

[8] Yang X, Ren Y, 2015, Advances in Pharmacological Action of Carthamin Yellow. Journal of Tropical Medicine, 15(3): 421-424. 